\title{
Bin Picking Success Rate Depending on Sensor Sensitivity
}

\author{
Petr Dolezel \\ Faculty of Electrical \\ Engineering and Informatics \\ University of Pardubice \\ Pardubice, Czech Republic \\ petr.dolezel@upce.cz
}

\author{
Jan Pidanic \\ Faculty of Electrical \\ Engineering and Informatics \\ University of Pardubice \\ Pardubice, Czech Republic \\ jan.pidanic@upce.cz
}

\author{
Tomas Zalabsky \\ Faculty of Electrical \\ Engineering and Informatics \\ University of Pardubice \\ Pardubice, Czech Republic \\ tomas.zalabsky@upce.cz
}

\author{
Miroslav Dvorak \\ Faculty of Electrical \\ Engineering and Informatics \\ University of Pardubice \\ Pardubice, Czech Republic \\ miroslav.dvorak@upce.cz
}

\begin{abstract}
The goal of this contribution is to determine correlation between an applied sensor for object registration and the success rate of the bin-picking problem. In most applications of a bin picking problem in industry, the procedure consists of two consecutive steps. The first step provides an initial guess of both position and rotation angle of the object to be registered, while the second one improves the exact pose accuracy, as required in following tasks. The second step can be, among others, implemented by the Iterative Closest Point Algorithm (ICP). It is well known that the ICP algorithm is very sensitive to the initial guess of the position and rotation angle of the object. Another interesting feature, especially from the technicians point of view, is the sensitivity of the ICP algorithm in relation to the applied sensor. Therefore, one particular bin picking application, involving complex irregular objects, is examined in this paper. Various kinds of sensors for 3D scene reconstruction are employed and, as a result of this contribution, a comprehensive set of relations between sensor quality and the ICP algorithm sensitivity is formulated.
\end{abstract}

Keywords-Bin Picking; Point Clouds; Pose Estimation; Robotic Arm.

\section{INTRODUCTION}

With the increasing interest in regards to industrial automation, many results of fundamental research related to robotics are being applied to industrial production. The automotive industry is a great example of this procedure [1], since this sector is irrepressibly forced into automation by both great demand for products and lack of adequate manpower. Many problems, that are not particularly interesting from an academic point of view, arise as a natural result of automation. On the other hand, the mentioned problems are very relevant for engineers, who implement the systems of automation into assembly lines.

The bin picking problem is, without any question, one of the typical instances. Although a theoretically solved issue, it still offers many practical problems to deal with [2]. Generally, a bin picking problem solution provides a robotic arm(s) capable of grabbing a particular object and placing it in a defined position. It should include any initial position and rotation angle of the object as well as possible obstacles and barriers in an operating area. A very detailed survey of bin picking implementation and applications can be found in [3].
This contribution deals with the first challenge of the bin picking problem chain. In order for successful performance, the object of interest has to be precisely registered, i.e. its position and rotation angle of the particular object has to be estimated among other objects, considering various limitations. Many different techniques have been proposed during the last decades [4], [5], [6]. Almost every proposed approach uses some sort of computer vision. However, due to an increasing availability of laser scanners, point clouds are more frequently being implemented for object registration [7]. Consequently, a new set of registration algorithms becomes available, Iterative Closest Point (ICP) algorithm being one of them [8].

One of the crucial features of the ICP algorithm is its sensitivity to an initial guess of a position and rotation angle of the registered object [9]. Apparently, the sensitivity rate depends on an immense set of features, including size and complexity of the object or the resolution of the point cloud [10]. Thus, it is not possible to analytically determine the required initial guess accuracy for arbitrarily shaped objects of interest. Therefore, a sensitivity rate relevant to the particular conditions of the application should be estimated in order to develop a robust bin picking application.

This contribution follows the article published in [11], where the authors investigate the relationship between the initial guess accuracy and the ICP algorithm convergence for one particular bin picking application, involving a PhoXi 3D laser scanner as a source of point cloud. The Phoxi 3D scanner family of laser scanners by Photoneo is a cutting edge set of sensors designed directly for bin picking. However, acquisition and installation costs are enormous compared with less accurate, but much cheaper 3D sensors. Therefore, the goal of this contribution is to determine the correlation between an applied sensor for object registration and the success rate of the bin-picking problem. Still, the investigation is performed in the framework of [11], i.e. the same bin picking problem is considered.

The paper is structured as follows. The problem is formulated in Section II. Then, a set of experiments suitable for the problem solution process is introduced in Section III and the 


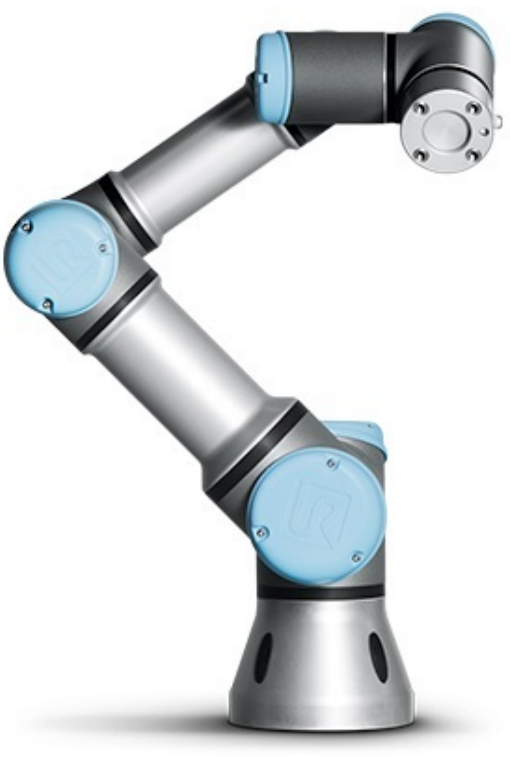

Fig. 1: UR3 by Universal Robots.

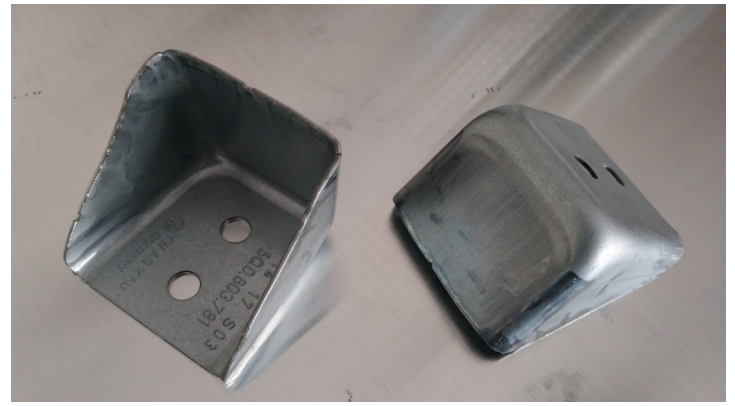

Fig. 2: Object of interest.

results are discussed in Section IV. Eventually, the paper is concluded in Section V.

\section{PRoblem FORMULATION}

A long-term contractor of the University of Pardubice asked for the improvement of an existing pick and place system. A UR3 type robotic arm by Universal Robots (see Fig. 1) is used to pick an object of interest and place it to a defined position for subsequent processing.

The aim of the setting is to find a position of an optimally located object (see Fig. 2) in a box of scattered pieces (see Fig. 3), grab it and move to a desired position. The object registration, as a part of the pick and place procedure, is used as follows [11].

- Get the 3D point cloud of the object of interest and the 3D point cloud of the scene as the inputs to the procedure;

- get the initial guess of the optimal position and rotation angle;

- use the ICP algorithm for iterative accurate registration of the object.

The ICP algorithm is an iterative method to align two objects using point clouds. Hence, if the position and rotation

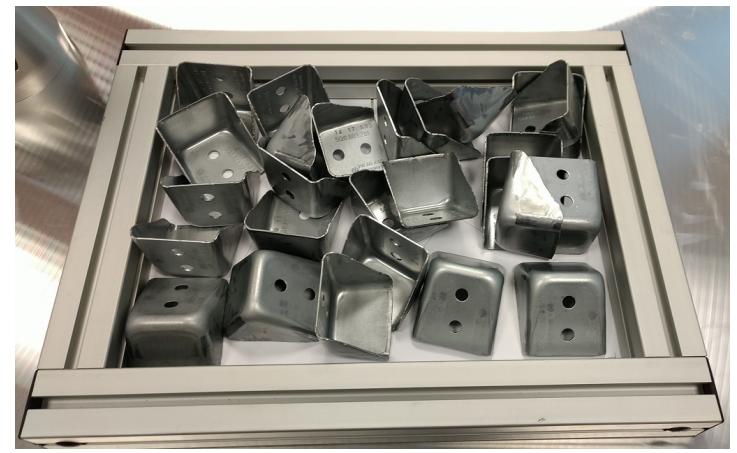

Fig. 3: Bin with components.

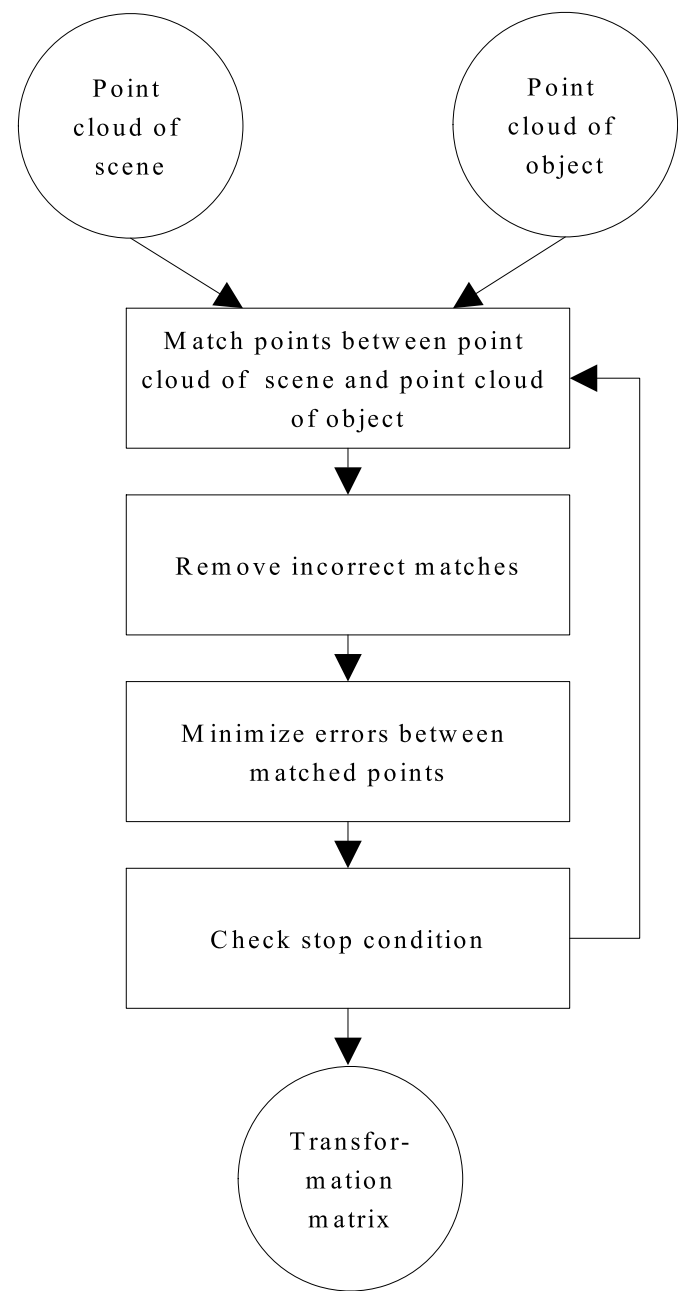

Fig. 4: ICP algorithm.

angle of one point cloud is set, it iteratively searches for a particular operation composed of translation and rotation, which transforms the other point cloud to the pose which minimizes differences between each couple of corresponding points found in both point clouds. The process of the ICP algorithm is depicted in Fig. 4, see [8] for a detailed description of each step. Practically speaking, the aim is to find a compromise between the accuracy and the acquisition cost of the 3D sensor.

Since the ICP is the iterative algorithm, an initial guess is 


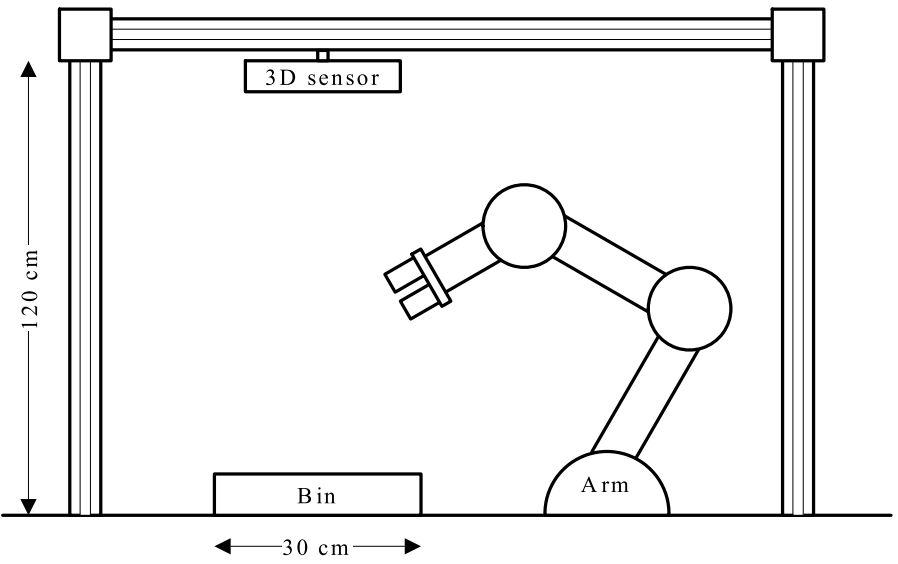

Fig. 5: Settings of experiment.

required to be set. Much like other iterative methods, the ICP algorithm can diverge with an inappropriately selected initial guess. The safe distance (uncertainty) of the initial guess in both position and rotation angle, suitable enough for the ICP algorithm to converge, is dealt with in [11]. However, just one type of 3D sensor is considered in the cited source. Therefore, the idea to be examined in this contribution is the affect of the safe uncertainty of the initial guess on the quality and features of the 3D sensor. In the engineering point of view, the goal is to find sufficiently accurate 3D sensor, which is simultaneously as low-cost as possible.

Thus, a set of experiments is performed in order to achieve the goal. In the following subsections, the particular setting of the pick and place system is described. Then, the list of possible 3D sensors is provided and, finally, the set of experiments to accomplish the goal is defined.

\section{EXPERIMENTS}

\section{A. Setting of the system}

An experimental stand is prepared to emulate the situation in an industrial environment. It consists of a UR3 robotic arm with a payload of $3 \mathrm{~kg}$, a 360-degree rotation on all wrist joints, and an infinite rotation on the end joint. Its reach exceeds $50 \mathrm{~cm}$. Moreover, the stand is equipped with a 3D sensor situated above the scene. A bin with the objects of interest is located near the base of the robotic arm. The floor dimensions of the bin are $45 \times 30 \mathrm{~cm}$, the maximum thickness of the layer of objects is $10 \mathrm{~cm}$. The setting is shown in Fig. 5 .

\section{B. Possible $3 D$ sensors}

Three types of 3D sensors, which are currently available for testing, are considered as possible sources of 3D point clouds.

1) Phoxi 3D scanner L: Phoxi 3D scanners by Photoneo are 3D scanners based on the parallel structured light. PhoXi 3D Scanner $\mathrm{L}$ is able to scan the volume of $110 \times 80 \times 90 \mathrm{~cm}$ with point to point distance less than $0.6 \mathrm{~mm}$. The standard price of PhoXi 3D Scanner L according to the price list is $12,450.00 €$. More information can be found in [12].

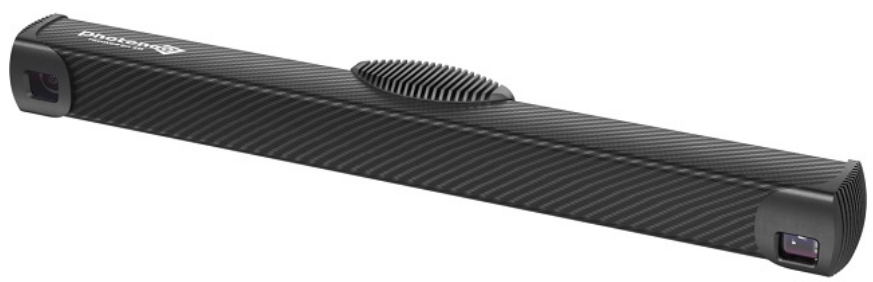

Fig. 6: Phoxi 3D scanner L.

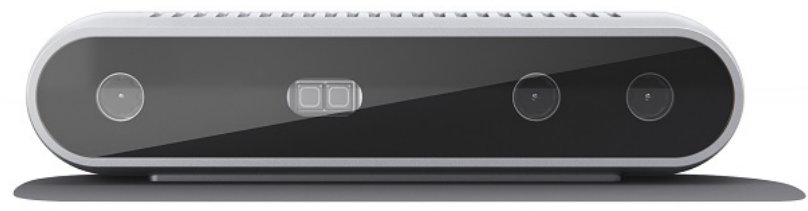

Fig. 7: Intel RealSense Depth Camera D415.

2) Intel RealSense Depth Camera D415: Intel RealSense technologies provide computer vision capabilities by equipping devices with the ability to see, understand, interact with, and learn from their environment. The Intel RealSense Depth Camera D415 is based on Active IR Stereo technology. It provides up to $1280 \times 720$ active stereo depth resolution with minimum depth distance of $16 \mathrm{~cm}$ and maximum range more than $10 \mathrm{~m}$. The frame rate is up to $90 \mathrm{fps}$. Its price starts at $208.00 €$ according to a worldwide comparison of e-shops. More information is available in [13].

3) Microsoft Kinect v2 sensor: Microsoft Kinect family of devices is a well-known group of sensors especially due to their application for home gaming systems. However, it has been applied for many other purposes, too. Kinect v2 sensor is based on Infra Red Time of Flight principle. It provides $512 \times 424$ pixels resolution with a field of view $70 \times 60$ degrees and 30 frames per second. Operative measuring range is from 0.5 to $4.5 \mathrm{~m}$. Microsoft Kinect can be acquired for less than $200 €$ according to the worldwide e-shops comparison. It is, however, no longer produced and is expected to be replaced with a new version. More information can be found in [14].

For a preliminary comparison, the point clouds acquired by all the sensors are depicted in Figs. 9, 10 and 11. The figures demonstrate the accuracy of the point clouds, which should illustrate the shape of the object of interest. Apparently, there are big differences between the qualities of the sensors.

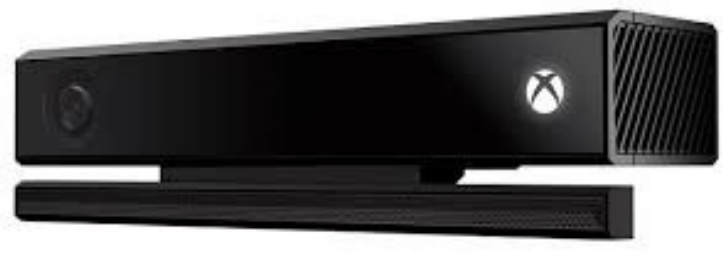

Fig. 8: Microsoft Kinect v2 sensor. 


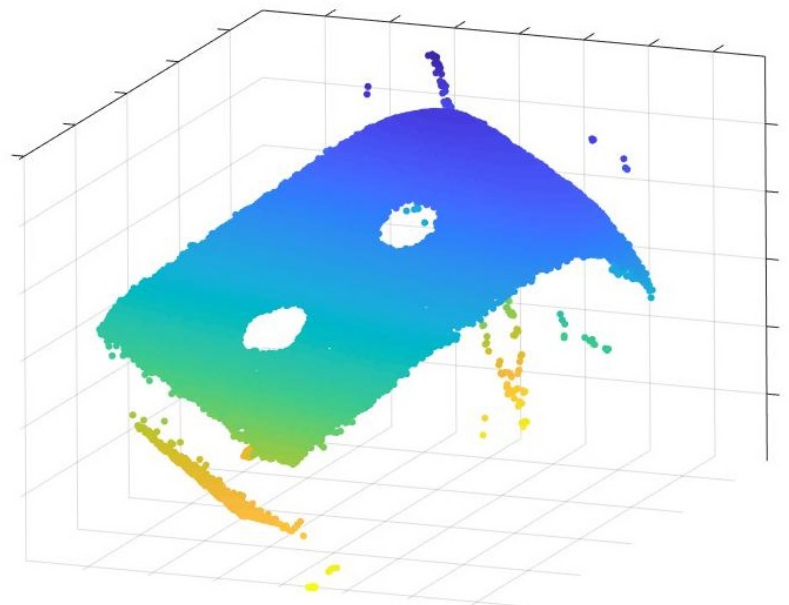

Fig. 9: Point cloud of object of interest gained using Phoxi 3D scanner L (172800 points).

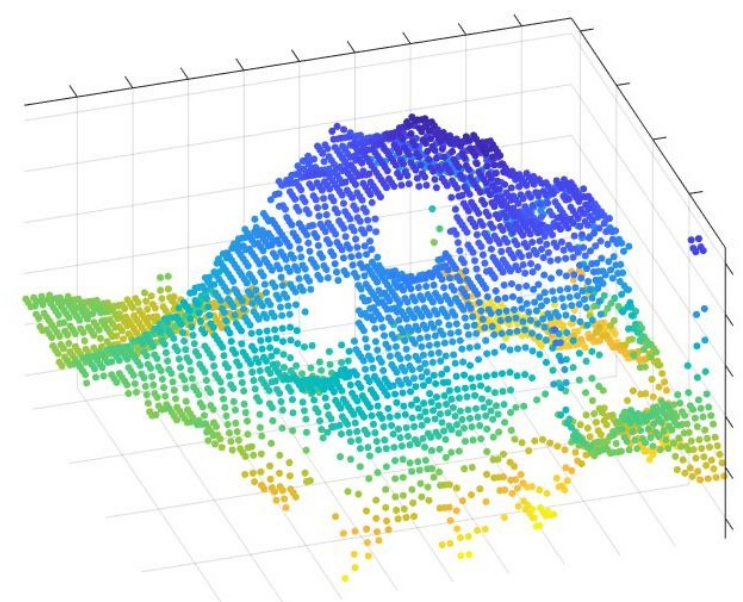

Fig. 10: Point cloud of object of interest gained using Intel RealSence Depth Camera D415 (3271 points).

\section{Settings of experiments}

A comprehensive set of many scenarios has been performed in order to achieve enough information for the suitable $3 \mathrm{D}$ sensor selection. In the following paragraphs, three types of Monte Carlo testing scenarios are presented, since, according to the authors' opinion, they are considered as the most representative ones.

\section{1) Scenario 1:}

- The position and rotation angle of the object of interest in a bin is known;

- The initial guess is put to a random position according to the following rules:

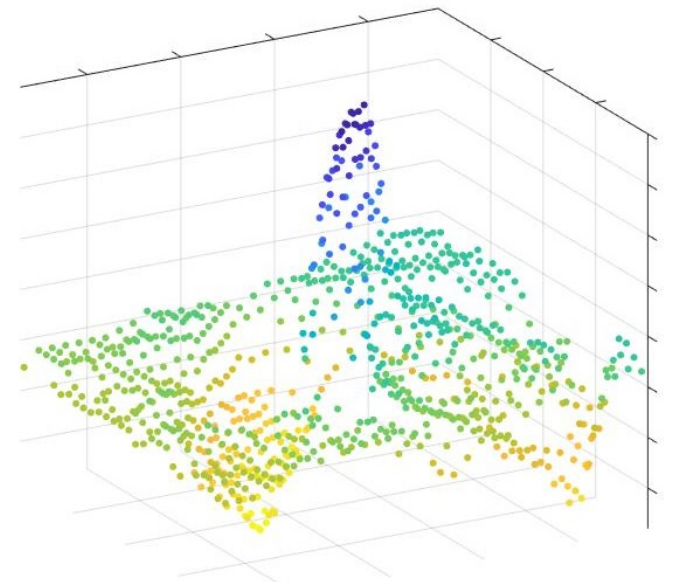

Fig. 11: Point cloud of object of interest gained using Microsoft Kinect v2 sensor (7434 points).

- The rotation angle $\alpha$ of the initial guess is equal to the rotation angle of the object of interest;

- The $\mathrm{x}, \mathrm{y}$ and $\mathrm{z}$ coordinates of the initial guess are moved to a random position relative to the position of the object of interest. The maximum distance in the $x$ and y coordinates is $200 \mathrm{~mm}$, in the $\mathrm{z}$ coordinate 100 $\mathrm{mm}$, uniform distribution of probability is applied;

- The ICP algorithm for iterative accurate registration of the object is used.

2) Scenario 2:

- The position and rotation angle of the object of interest in a bin is known;

- The initial guess is put to a random position according to the following rules:

- The $\mathrm{x}, \mathrm{y}$ and $\mathrm{z}$ coordinates of the initial guess are equal to the position of the object of interest;

- The rotation angle $\alpha$ of the initial guess is set to a random value between $0^{\circ}$ and $90^{\circ}$ from the rotation angle of the object of interest. Uniform distribution of probability is applied. Counter-clockwise rotation is defined using the right hand rule in reference to the direction vector $u=\left[u_{1}, u_{2}, u_{3}\right]$, where each element of the vector $u$ is generated randomly using the uniform distribution, $u_{i} \in[0,1]$.

- The ICP algorithm for iterative accurate registration of the object is used.

3) Scenario 3:

- The position and rotation angle of the object of interest in a bin is known;

- The initial guess is put to a random position according to the following rules:

- The $\mathrm{x}, \mathrm{y}$ and $\mathrm{z}$ coordinates of the initial guess are moved to a fixed position relative to the position of 
the object of interest. The position in the $\mathrm{x}$ and $\mathrm{y}$ coordinates is moved by $150 \mathrm{~mm}$, in the $\mathrm{z}$ coordinate by $100 \mathrm{~mm}$;

- The rotation angle $\alpha$ of the initial guess is set to $75^{\circ}$ from the rotation angle of the object of interest. Counter-clockwise rotation is defined using the right hand rule in reference to the direction vector $u=$ $\left[u_{1}, u_{2}, u_{3}\right]$, where each element of the vector $u$ is generated randomly using the uniform distribution, $u_{i} \in[0,1]$.

- The ICP algorithm for iterative accurate registration of the object is used.

For all experiments, 10000 samples are applied in order to achieve a statistically significant number of data. The parameters of the ICP algorithm are set as follows: the maximum number of iterations is set to 1000 epochs and the stopping criterion is defined as the 2-element vector $\delta=\left[\delta_{1}, \delta_{2}\right]=[0.001,0.01]$, that represents the absolute difference in translation and rotation estimated in two consecutive iterations. $\delta_{1}$ measures the Euclidean distance between two translation vectors, $\delta_{2}$ represents the angular difference in degrees.

\section{RESULTS OF EXPERIMENTS}

1) Scenario 1: This testing scenario is performed using all three sensors and settings shown in Fig. 5. The ratio of correctly registered objects of interest against the number of samples (success rate) is examined. In addition, more detailed features are determined, too. Namely, the correlation of the success rate and the absolute distance $D$ between the object of interest and initial guess position.

TABLE I: Success rate according to the scenario 1

\begin{tabular}{|l|c|c|c|c|}
\cline { 2 - 5 } \multicolumn{1}{c|}{} & \multicolumn{4}{c|}{ Success rate } \\
\hline Sensor & Overall & $D<5 \mathrm{~cm}$ & $D<10 \mathrm{~cm}$ & $D<20 \mathrm{~cm}$ \\
\hline Phoxi 3D scanner L & 0.7076 & 0.9765 & 0.8613 & 0.7384 \\
\hline Intel RealSense D415 & 0.6817 & 0.9663 & 0.8345 & 0.7188 \\
\hline Microsoft Kinect v2 & 0.0608 & 0.0816 & 0.0643 & 0.0628 \\
\hline
\end{tabular}

Considering the results in Table I as well as the evident Fig. 11, Microsoft Kinect v2 is obviously ineligible for this particular application. However, both remaining sensors provide surprisingly similar results.

\section{A. Scenario 2}

This scenario is performed using Phoxi 3D scanner $\mathrm{L}$ and RealSense D415, since Microsoft Kinect v2 was excluded after scenario 1 testing. The overall success rate, as well as the success rates in correlation to the maximal absolute rotation angle are summarized in Table II.

TABLE II: Success rate according to the scenario 2

\begin{tabular}{|l|c|c|c|c|}
\cline { 2 - 5 } \multicolumn{1}{c|}{} & \multicolumn{4}{c|}{ Success rate } \\
\hline Sensor & Overall & $\alpha<45^{\circ}$ & $\alpha<60^{\circ}$ & $\alpha<75^{\circ}$ \\
\hline Phoxi 3D scanner L & 0.9504 & 1.0000 & 1.0000 & 0.9988 \\
\hline Intel RealSense D415 & 0.9306 & 1.0000 & 1.0000 & 0.9943 \\
\hline
\end{tabular}

\section{B. Scenario 3}

This scenario is performed using Phoxi 3D scanner $\mathrm{L}$ and RealSense D415. The ratio of correctly registered objects of interest against the number of samples is again examined. This experiment considers both translation and rotation, while the rotation direction is variable. The results are shown in Table III.

TABLE III: Success rate according to the scenario 3

\begin{tabular}{|l|c|}
\hline Sensor & Overall success rate \\
\hline Phoxi 3D scanner L & 0.6676 \\
\hline Intel RealSense D415 & 0.5643 \\
\hline
\end{tabular}

\section{CONCLUSION}

An accurate registering of defined complex objects, depending on the sensor for 3D scene reconstruction, was dealt with in the paper. It is necessary to emphasize, that the results achieved in the experiments presented above are strongly connected to the specific setting demanded by a contracting authority. However, the findings can be roughly generalized into a category of similar situations. Particularly, a precise registration of complex components is significantly dependent on the quality of the 3D sensor. Nevertheless, the dependency is far from being linear. While the Phoxi 3D scanner provided the most accurate data, it turned out, that for our particular application, the Intel RealSense D415 camera provided suitable enough information, too. Apparently, the resolution of the point cloud provided by the RealSense camera was limited. However, it was accurate enough for the ICP algorithm to achieve results close to the results gained with the Phoxi RealSense sensor. Microsoft Kinect v2, on the other hand, proved itself to be absolutely ineligible for this situation.

Generally, the absolute tolerance of an initial guess uncertainty for the ICP algorithm needs to be known in order to achieve the overall stability of the bin picking problem solution. It is affected by the shape of the object of interest, its material, light condition and many other features. Thus, for industrial applications, it is still necessary to perform many testing experiments (like those mentioned above) apart from an analytic approach.

\section{ACKNOWLEDGMENT}

The work was supported from ERDF/ESF "Cooperation in Applied Research between the University of Pardubice and companies, in the Field of Positioning, Detection and Simulation Technology for Transport Systems (PosiTrans)" (No. CZ.02.1.01/0.0/0.0/17_049/0008394).

\section{REFERENCES}

[1] H. Ghafil and K. Jrmai, "Research and application of industrial robot manipulators in vehicle and automotive engineering, a survey," Lecture Notes in Mechanical Engineering, no. 9783319756769, pp. 611-623, 2018.

[2] M. Alonso, A. Izaguirre, and M. Graa, "Current research trends in robot grasping and bin picking," Advances in Intelligent Systems and Computing, vol. 771, pp. 367-376, 2019. 

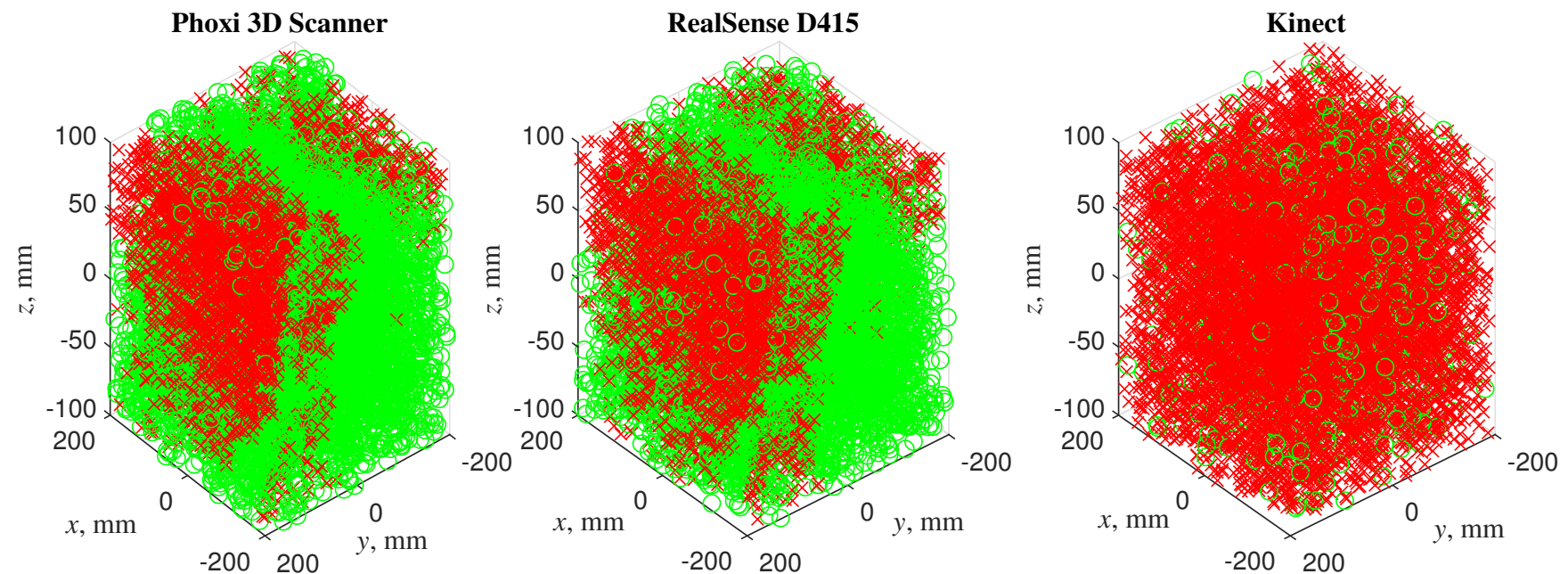

Fig. 12: Graphical representation of scenario 1 results. Green circles represent successful attempts while red crosses mean unsuccessful attempts.

[3] A. Pochyly, T. Kubela, V. Singule, and P. Cihak, "3d vision systems for industrial bin-picking applications," in Proceedings of 15th International Conference MECHATRONIKA, Dec 2012, pp. 1-6.

[4] H. Y. Kuo, H. R. Su, S. H. Lai, and C. C. Wu, "3d object detection and pose estimation from depth image for robotic bin picking," in 2014 IEEE International Conference on Automation Science and Engineering (CASE), Aug 2014, pp. 1264-1269.

[5] M. Ulrich, C. Wiedemann, and C. Steger, "Combining scale-space and similarity-based aspect graphs for fast $3 \mathrm{~d}$ object recognition," IEEE Transactions on Pattern Analysis and Machine Intelligence, vol. 34, no. 10, pp. 1902-1914, Oct 2012.

[6] K. Rahardja and A. Kosaka, "Vision-based bin-picking: recognition and localization of multiple complex objects using simple visual cues," in Intelligent Robots and Systems '96, IROS 96, Proceedings of the 1996 IEEE/RSJ International Conference on, vol. 3, Nov 1996, pp. 1448-1457 vol.3.

[7] D. Holz, A. E. Ichim, F. Tombari, R. B. Rusu, and S. Behnke, "Registration with the point cloud library: A modular framework for aligning in 3-d," IEEE Robotics Automation Magazine, vol. 22, no. 4, pp. 110-124, Dec 2015.

[8] P. J. Besl and N. D. McKay, "A method for registration of 3-d shapes," IEEE Transactions on Pattern Analysis and Machine Intelligence, vol. 14, no. 2, pp. 239-256, Feb 1992.

[9] J. K. Oh, C. H. Lee, S. H. Lee, S. H. Jung, D. Kim, and S. Lee, "Development of a structured-light sensor based bin-picking system using icp algorithm," in ICCAS 2010, Oct 2010, pp. 1673-1677.

[10] M. Attia and Y. Slama, "Efficient initial guess determination based on 3d point cloud projection for icp algorithms," in 2017 International Conference on High Performance Computing Simulation (HPCS), July 2017, pp. 807-814.

[11] J. Tvrdik and P. Dolezel, "Fine localization of complex components for bin picking," Advances in Intelligent Systems and Computing, vol. 868, pp. 246-255, 2019.

[12] Phoxi 3D scanners by Photoneo, 2019 (accessed February 1, 2019), https://www.photoneo.com/products/phoxi-scan-1/.

[13] Introducing Intel RealSense Depth Cameras, 2019 (accessed February 1, 2019), https://realsense.intel.com/depth-camera/.

[14] E. Lachat, H. Macher, T. Landes, and P. Grussenmeyer, "Assessment and calibration of a rgb-d camera (kinect v2 sensor) towards a potential use for close-range 3d modeling," Remote Sensing, vol. 7, pp. 13070 $13097,2015$. 